\title{
Fast 3D Microscopy Imaging of Contacts Between Surfaces Using a Fluorescent Liquid
}

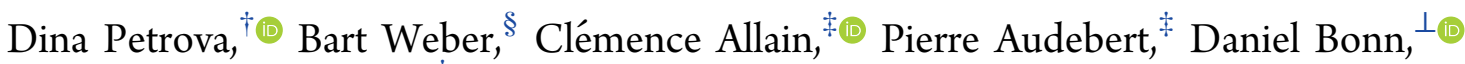 \\ and Albert M. Brouwer* ${ }^{* \dagger} \odot$ \\ $\dagger$ van 't Hoff Institute for Molecular Sciences, University of Amsterdam, Amsterdam P.O. Box 94157, 1090 GD, The Netherlands \\ ${ }^{\S}$ Advanced Research Center for Nanolithography (ARCNL), Amsterdam P.O. Box 93019, 1090 BA, The Netherlands \\ ${ }^{\ddagger}$ PPSM, ENS Cachan, CNRS, Université Paris-Saclay, Cachan 94235, France \\ ${ }^{\perp}$ Institute of Physics, University of Amsterdam, Amsterdam P.O. Box 94485, 1090 GL, The Netherlands
}

\section{Supporting Information}

ABSTRACT: A novel method is presented for the rapid direct $3 \mathrm{D}$ visualization of the contact between two surfaces by means of fluorescence microscopy using a fluorescent liquid. Distances between the surfaces of up to several hundred nanometers can be determined with subnanometer accuracy in $3 \mathrm{D}$ and within seconds of measurement time. The method opens new possibilities for research in the areas of contact mechanics, friction, wear, and lubrication.

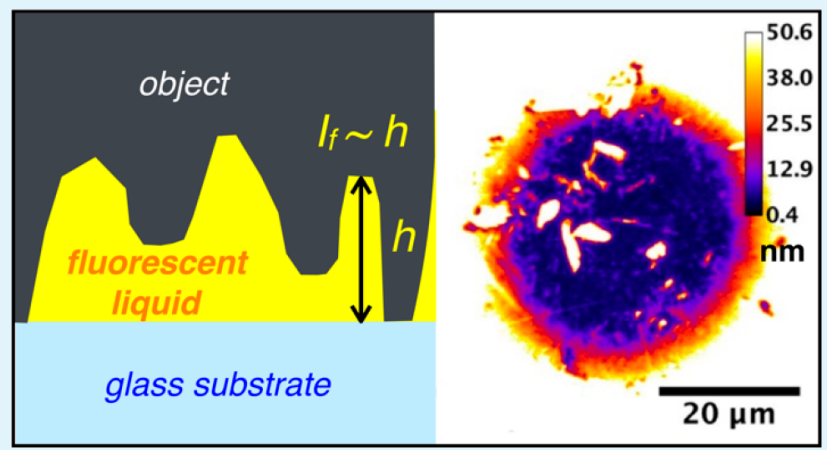

KEYWORDS: interfaces, mechanical properties, fluorescent probes, 3D imaging

$\mathrm{E}$ arthquakes, ${ }^{1}$ tire grip, ${ }^{2}$ engine losses, ${ }^{3}$ precision positioning, ${ }^{4}$ and bearings ${ }^{5}$ are all examples of phenomena, processes or objects in which friction plays a crucial role. In these examples, macroscopically applied normal and tangential forces lead to stresses that may approach or exceed the elastic limit at the microscopic contacts that define the interface between rough surfaces. Deformation, slip, and wear mechanisms that occur under these extreme conditions remain elusive because few experiments provide in situ observation at the appropriate length scales. In this letter, we present a new method for quantitative imaging of mechanical contacts, making use of a low viscosity fluorescent liquid.

Measurement of the 3D topography of a surface can be done with imaging methods such as atomic force microscopy (AFM), scanning tunneling microscopy (STM), and scanning electron microscopy (SEM). However, these techniques do not enable visualization of surface deformations caused by contact with another object and additionally have the disadvantage that they may change the material properties on small scales while probing the sample with either a sharp tip or an electron beam. Optical methods, such as fluorescencebased digital imaging correlation (FDIC), ${ }^{6-9}$ fluorescence stereo microscopy, ${ }^{9,10}$ fluorescence-interferometry ${ }^{11}$ and labelfree SGH generation ${ }^{12}$ can be used for imaging two surfaces simultaneously, but they have their drawbacks such as Abbe's diffraction limit in all dimensions, small fields of view, long acquisition times, poor resolution, or complicated mathemat- ical postprocessing. ${ }^{6,13}$ White-light interferometry is one of the most precise techniques for the measurement of surface profiles, but its axial resolution decreases in case of steep surfaces. ${ }^{14-17}$ In addition to these methods being applicable mainly for measurements in $2 \mathrm{D}$, they also have drawbacks such as a low speed of acquisition and the need for high-speed cameras, which makes these methods costly.

Here, we introduce a novel, cost-efficient method for the direct rapid $3 \mathrm{D}$ visualization of the contact between an optically transparent substrate and an arbitrary object. By using a fluorescent liquid and confocal fluorescence microscopy, subnanometer accuracy is obtained in the axial direction. With a simple calibration the method can be applied as a fast and quantitative method to visualize the $3 \mathrm{D}$ profile and contacts between two surfaces.

To achieve this, we place a smooth flat glass coverslip on an inverted confocal microscope coupled to a rheometer for mechanical measurements. ${ }^{19,20}$ On the coverslip we deposit a droplet of a fluorescent liquid, ${ }^{18}$ a liquid of which every single molecule is fluorescent (Scheme 1). ${ }^{21}$ A sphere attached to a plate geometry mounted on the rheometer is lowered into contact with the wetted coverslip, and the contact plane is illuminated from below. The molecules of fluorescent liquid

Received: September 9, 2018

Accepted: November 15, 2018

Published: November 15, 2018 
Scheme 1. Molecular Structure of the Fluorescent Liquid 3,6-Bis((2-ethylhexyl)oxy)-1,2,4,5-tetrazine $1 .^{18}$

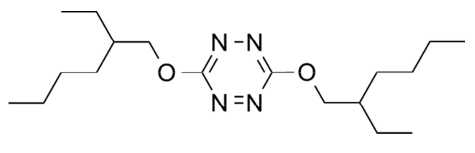

present at the interface are excited by laser light and their fluorescence is captured-also from below - by the confocal microscope. By scanning in the contact plane, a $3 \mathrm{D}$ intensity image is obtained because the intensity of the fluorescence in each image pixel is proportional to the number of fluorescent molecules in the excited volume. Thus, the signal is directly proportional to the length of the light path, that is, the gap between the surfaces.

As all the molecules of the liquid are fluorescent, the gap between two surfaces can be determined with a precision of one molecular layer, corresponding to subnm precision. By determining the proportionality between the thickness and the intensity, it is possible to quantitatively reconstruct the $3 \mathrm{D}$ structure of the interface from the consecutive $2 \mathrm{D}$ intensity images.

A number of requirements must be met for our simple approach to work. First of all, we approximate the confocal point spread function as a cylinder, in which the excitation light intensity is nearly constant. This approximation is valid if the layer of liquid is not more than a few hundred nm thick, and if the light is not strongly attenuated by absorption. We calculate the attenuation of the laser intensity using the Lambert-Beer law. ${ }^{22}$ Taking into account the molar absorption coefficient at the laser excitation wavelength of $514 \mathrm{~nm} \varepsilon=630 \mathrm{M}^{-1} \mathrm{~cm}^{-1}$, 19 and the concentration of neat liquid $c=4.4 \mathrm{M}$, we calculate that an attenuation of the light intensity by $1 \%$ occurs over a light path of $36 \mathrm{~nm}$. At a height of $344 \mathrm{~nm}$, the intensity is still $90 \%$ of the original intensity. Because the emission is detected in a wavelength range that does not overlap with the absorption spectrum we can neglect attenuation of the emitted light by reabsorption. Deviations from this ideal situation can be tested experimentally as described below.

In Figure 1, the measured fluorescence intensity crosssection through the center of a glass precision sphere gently pressed onto the coverslip is shown. The center part of the sphere is flattened at the contact due to elastic deformation, quantitatively described by Hertz' theory, ${ }^{23}$ and the surface curvature of the sphere outside the contact is modeled using a second-order polynomial.

To obtain a scaling factor between the intensity of the fluorescence and the thickness of the liquid,we performed a calibration using the image of the reflection of the laser from the contact between two surfaces. The reflections form a pattern of dark and bright rings-also known as Newton rings $-{ }^{24,25}$ due to the interference of the laser light reflected off the sphere/liquid interface with that reflected off the liquid/ coverslip interface. These rings occur when the gap between the surfaces $d$ is equal to

$$
d=\left(m+\frac{1}{2}\right) \frac{\lambda}{2 n}
$$

where $m=0,1,2,3, \ldots$, is the ring number, $\lambda=514 \mathrm{~nm}$ is the wavelength of the light, and $n=1.483$ is the refractive index of the fluorescent liquid. Thus, by plotting fluorescence intensity values versus the heights obtained from eq 1 , a scaling factor is

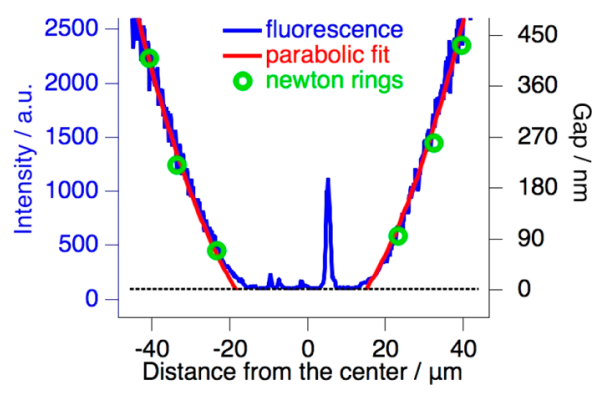

Figure 1. Fluorescence intensity cross-section through the center of the interface between a $3.175 \mathrm{~mm}$ diameter glass sphere and a smooth glass coverslip filled with fluorescent liquid 1 (blue line). Intensities are converted to $\mathrm{nm}$ units as described in the text (right axis). The applied normal force on the sphere is $100 \mathrm{mN}$. The fluorescence intensity is fitted with a second-order polynomial (red line) corresponding to the shape of the sphere. At the contact the sphere is flattened due to application of the normal force. The green circles designate the positions of the Newton rings in the interference pattern recorded simultaneously. As explained in the text, these rings are used to convert the intensity units to nanometers. The horizontal dashed line represents the dark count level of the detector.

obtained (see Figures S1-S3). Importantly, the reflection mode is measured simultaneously with fluorescent mode on another detector, so the sample stays in the same position.

Fluorescence intensity images of the sphere surface in contact with the glass slide are presented in Figure 2. The scale
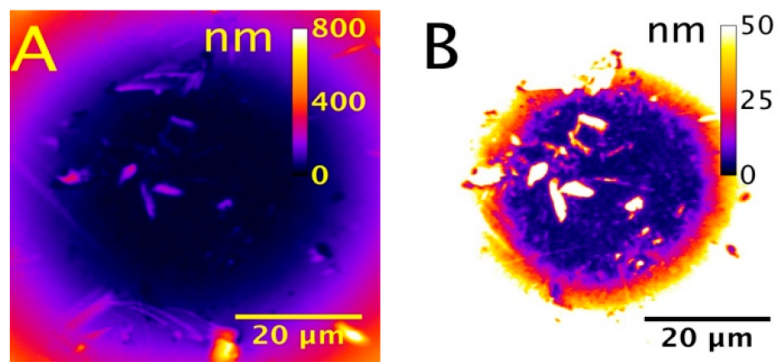

Figure 2. Fluorescence intensity images of a rough glass sphere on smooth glass interface immersed in the fluorescent liquid. A contact force of $100 \mathrm{mN}$ is applied using the rheometer. Intensities are converted to $\mathrm{nm}$ units. Different intensity scales are used in order to visualize different gap size ranges: (A) First $800 \mathrm{~nm}$ between the surfaces. (B) First $50 \mathrm{~nm}$ between the surfaces

bar represents height in $\mathrm{nm}$. The detector gain and the laser power were chosen in such a way that the dynamic range of the detector is used fully. The maximum intensity of $\sim 4000$ arbitrary units corresponds to a gap of $\sim 1 \mu \mathrm{m}$. The dark count level of the detector corresponds to 50 intensity units, which was subtracted from the overall image. We estimate the approximate thickness of a single layer of fluorescent liquid molecules using the molar concentration and the density of the liquid resulting in $\sim 0.8 \mathrm{~nm}$.

By using a different intensity scale, our method can be used for visualization of different ranges of the gap size between the surfaces. As shown in Figure $2 \mathrm{a}, \mathrm{b}$, both molecularly thin and thick layers containing several hundreds of molecules can be visualized with a single data set.

In Figure $2 b$, to clearly see the smallest details of the contact, the full color scale is used to represent the range $0-50 \mathrm{~nm}$, with gaps $>50 \mathrm{~nm}$ all having the same (white) color. 
To confirm the results obtained with the imaging method, we image the exact same section on the sphere surface visualized in the microscopy experiments by AFM before performing the fluorescence microscopy. In Figure 3, we
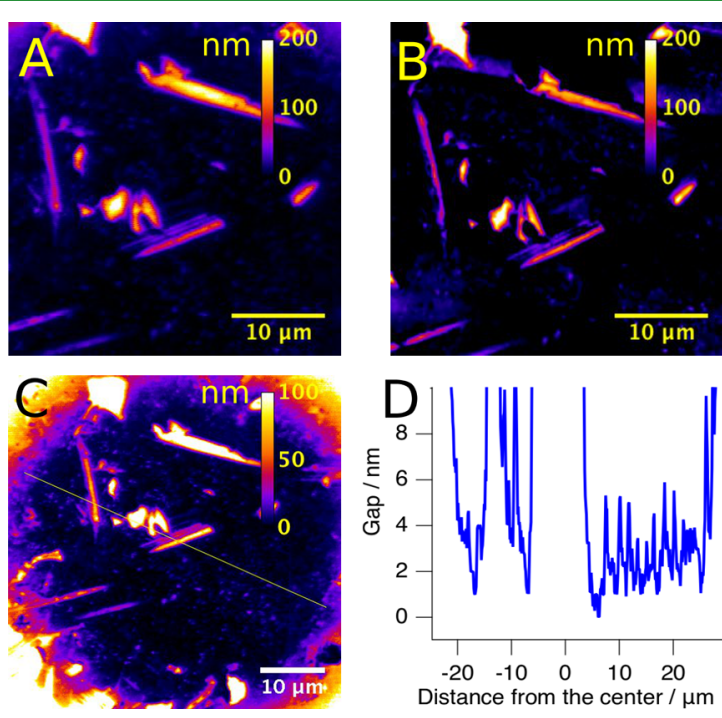

Figure 3. Interface between a smooth glass coverslip and a rough glass sphere. (A) Image obtained by using the fluorescent liquid method. The intensity scale is chosen such that gaps up to a few hundred nm are visible. (B) AFM image of the same glass sphere. (C) Crosssection (yellow line) on the image obtained with the fluorescent liquid method. (D) Cross-section of the image C. The scale is set to a few nanometers to demonstrate the sensitivity of the gap measurement. Noise level is $0.2 \mathrm{~nm}$.

compare the image obtained using the fluorescent liquid method (Figure 3a) with that obtained with AFM (Figure 3b; see also Figure S4). We set the dynamic range to a few hundred $\mathrm{nm}$. The comparison of the images shows that the $3 \mathrm{D}$ structure obtained using the fluorescent liquid method is the same within the experimental resolution as that obtained by AFM. Even the smallest features (1-2 nm height) can be observed in the fluorescence microscopy images (see Figure 3d). Moreover, molecular layers trapped between two surfaces are also resolvable (gap $>0$ at the contact plane, see Figure 3d).

Although the intensity calibration demonstrates the validity of our approach, it is important to investigate to what extent the photophysical properties of the fluorescent liquid are affected by the proximity of the two surfaces and by the contact pressure.

Emission spectra were measured at locations with different gaps between the glass sphere and the glass coverslip (Figure $4 a)$. We discriminate between regions where the gap $d$ between the surfaces is $d<10 \mathrm{~nm}$, regions with $d>10 \mathrm{~nm}$ and emission measured in the pure liquid. Spectra were smoothed to decrease the noise. The fluorescence spectra presented in Figure $4 \mathrm{a}$ show that there is only a small blue shift of the position of the emission maximum $(5-7 \mathrm{~nm})$ when the liquid is confined.

In addition, we performed time-correlated single photon counting (TCSPC) experiments to determine the fluorescence decay times of the liquid in our sphere-on-coverslip setup. We find that in the contact region the fluorescence lifetime is slightly reduced $(\sim 10 \%)$ compared to that measured in the pure liquid (Figure $4 \mathrm{~b}$ ), which could be related to the local

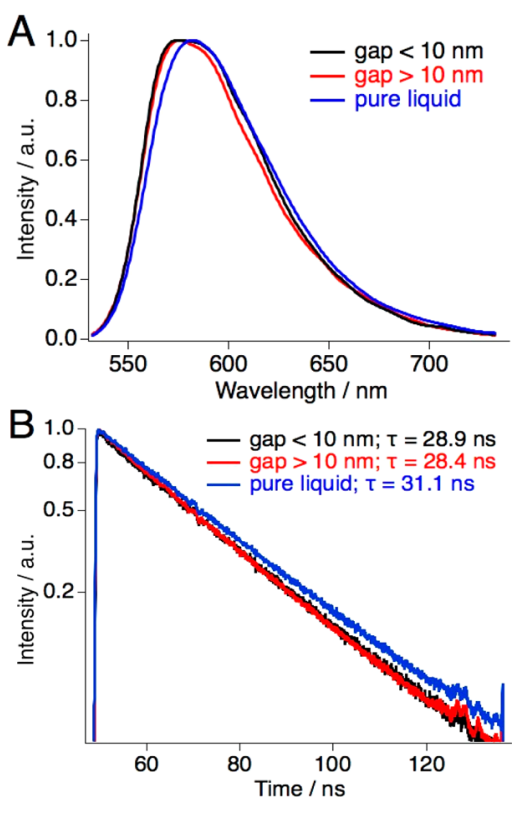

Figure 4. (A) Normalized fluorescence spectra of the liquid in "bright" and "dark" interface regions and in pure liquid between two glass surfaces. Each spectrum is an average of 100 spectra acquired at different locations of a particular type within the interface. (B) Comparison of fluorescence decay traces in "dark" contacts, "bright" contacts, and pure liquid.

pressure and confinement induced by the contacts. One of the special features of the tetrazine fluorescent liquid $\mathbf{1}$ is that the close contact between the molecules does not lead to substantial fluorescence quenching: the nonradiative decay rate is the same for the neat liquid and a dilute solution in dichloromethane. ${ }^{18}$ Overall, the photophysical properties of the fluorescent liquid do not depend substantially on the local gap. The detectable differences introduce only a small error, if any, to the measurement of the distance between the surfaces.

Our novel 3D contact imaging method readily allows for the quick visualization (within seconds) of micrometer gaps with subnm precision over areas up to $1 \times 10^{4} \mu \mathrm{m}^{2}$. The method can also be used with wide-field illumination because it is the presence of the fluorescent molecules in the observation volume that provides the measure of the layer thickness, and confocal sectioning is not essential. In this case, the acquisition time could be even smaller than with the scanning confocal microscope. The method can also be extended for the 3D visualization of surfaces with larger irregularities. For this purpose, the liquid can be diluted to reduce the absorption. For gaps in the range of several micrometers, regular $3 \mathrm{D}$ scanning confocal microscopy is more practical. While we have successfully applied the method to spheres made of glass and polystyrene, one of the current limitations is obviously the nature of the material, as it is required that the liquid stays between the two surfaces of interest and does not enter, for example, into a porous material, or cause swelling of a polymer surface. One should also be aware of potential fluorescence quenching pathways due to the presence of photoactive species at the surfaces, e.g., by heavy atom effects or excited-state electron transfer. ${ }^{26}$

Our method can be applied to study contact mechanics under loads in the range of hundreds of $\mathrm{MPa}$ (e.g., polystyreneon-glass ${ }^{19}$ ) to a few GPa (glass on glass ${ }^{19}$ ), as we showed that the photophysical properties of the fluorescent liquid are not 
affected significantly by the contact conditions (see Figure 4). As the fluorescence lifetime is somewhat shortened when the liquid is confined in smaller gaps, application of loads exceeding a few GPa might lead to underestimation of the thickness because the fluorescence quantum yield is reduced. At such high pressures, however, most of the liquid will be pressed out of the contact, and only nm thick wetting layers may remain, if any. Further study is needed to explore the limits of the application.

In summary, we introduce a novel method of imaging the contact between two surfaces in $3 \mathrm{D}$ by using a standard confocal fluorescence microscope and a fluorescent liquid. We showed that the fluorescence intensity in the image of the glass sphere is proportional to the $3 \mathrm{D}$ height profile obtained by AFM imaging. The proportionality factor between intensity and height can be determined by comparing the fluorescence with interference patterns. This is to our knowledge the first study to directly and quantitatively visualize the interfacial gap between two surfaces in 3D. It reveals information about deformation of the surface roughness, which is crucial in tribology. As our method can be used to determine the thickness of layers of liquid in between surfaces with subnm precision, it can be used for direct visualization and further understanding of lubrication and friction.

\section{ASSOCIATED CONTENT}

\section{S Supporting Information}

The Supporting Information is available free of charge on the ACS Publications website at DOI: 10.1021/acsami.8b15660.

Experimental details; determination of scaling factor via Newton rings; AFM vs fluorescence images for other materials (PDF)

\section{AUTHOR INFORMATION}

\section{Corresponding Author}

*E-mail: A.M.Brouwer@uva.nl. Phone: +31205255491.

\section{ORCID}

Dina Petrova: 0000-0002-6682-0760

Clémence Allain: 0000-0002-2908-264X

Daniel Bonn: 0000-0001-8925-1997

Albert M. Brouwer: 0000-0002-1731-3869

\section{Notes}

The authors declare no competing financial interest.

\section{ACKNOWLEDGMENTS}

This research was financially supported by The Netherlands Organization for the Advancement of Research (NWO) and by the European Research Council. Michiel Hilbers helped with the fluorescence decay time measurements.

\section{REFERENCES}

(1) Scholz, C. H. Earthquakes and Friction Laws. Nature 1998, 391, 37-42.

(2) Persson, B. N. J. Theory of Rubber Friction and Contact Mechanics. J. Chem. Phys. 2001, 115, 3840-3861.

(3) Holmberg, K.; Erdemir, A. Influence of Tribology on Global Energy Consumption, Costs and Emissions. Friction 2017, 5, 263284.

(4) Tomizuka, M. Robust Digital Motion Controllers for Mechanical Systems. Rob. Auton. Syst. 1996, 19, 143-149.

(5) Sander, D. E.; Allmaier, H.; Priebsch, H. H.; Witt, M.; Skiadas, A. Simulation of Journal Bearing Friction in Severe Mixed Lubrication -
Validation and Effect of Surface Smoothing due to Running-In. Tribol. Int. 2016, 96, 173-183.

(6) Hu, Z.; Xu, T.; Wang, X.; Xie, Z.; Luo, H.; He, Y.; Guo, L.; Li, Y.; Gan, R.; Lu, H. Fluorescent Digital Image Correlation Techniques in Experimental Mechanics. Sci. China: Technol. Sci. 2018, 61, 21-36.

(7) Ren, M.; Liang, J.; Li, L.; Wei, B.; Wang, L.; Tang, Z. Accurate Three-Dimensional Shape and Deformation Measurement at Microscale Using Digital Image Correlation. Rev. Sci. Instrum. 2015, 86, 075102 .

(8) Franck, C.; Hong, S.; Maskarinec, S. A.; Tirrell, D. A.; Ravichandran, G. Three-Dimensional Full-Field Measurements of Large Deformations in Soft Materials Using Confocal Microscopy and Digital Volume Correlation. Exp. Mech. 2007, 47, 427-438.

(9) Hu, Z.; Luo, H.; Du, Y.; Lu, H. Fluorescent Stereo Microscopy for 3D Surface Profilometry and Deformation Mapping. Opt. Express 2013, 21, 11808-11818.

(10) Hu, Z.; Luo, H.; Lu, H. Highly Accurate 3D Shape and Deformation Measurements Using Fluorescent Stereo Microscopy. In Advancement of Optical Methods in Experimental Mechanics; Springer International, 2016; Vol. 3, 85-93.

(11) Beltramo, P. J.; Vermant, J. Simple Optical Imaging of Nanoscale Features in Free-Standing Films. ACS Omega 2016, 1, 363-370.

(12) Jorge-Peñas, A.; Bové, H.; Sanen, K.; Vaeyens, M. M.; Steuwe, C.; Roeffaers, M.; Ameloot, M.; Van Oosterwyck, H. 3D Full-Field Quantification of Cell-Induced Large Deformations in Fibrillar Biomaterials by Combining Non-Rigid Image Registration with Label-Free Second Harmonic Generation. Biomaterials 2017, 136, 86-97.

(13) Ţălu, Ş. Micro and Nanoscale Characterization of Three Dimensional Surfaces: Basics and Applications; Napoca Star: ClujNapoca, Romania, 2015.

(14) Montgomery, P.; Anstotz, F.; Montagna, J. High-Speed, onLine 4D Microscopy Using Continuously Scanning White Light Interferometry with a High-Speed Camera and Real-Time FPGA Image Processing. Proc. SPIE 2011, 8082, 808210-808219.

(15) Liu, M.; Cheung, C. F.; Ren, M.; Cheng, C.-H. Estimation of Measurement Uncertainty Caused by Surface Gradient for a White Light Interferometer. Appl. Opt. 2015, 54, 8670-8677.

(16) Coupland, J. M.; Lobera, J. Measurement of Steep Surfaces Using White Light Interferometry. Strain 2010, 46, 69-78.

(17) Eguchi, M.; Shibamiya, T.; Yamamoto, T. Measurement of Real Contact Area and Analysis of Stick/Slip Region. Tribol. Int. 2009, 42, $1781-1791$.

(18) Allain, C.; Piard, J.; Brosseau, A.; Han, M.; Paquier, J.; Marchandier, T.; Lequeux, M.; Boissière, C.; Audebert, P. Fluorescent and Electroactive Low-Viscosity Tetrazine-Based Organic Liquids. ACS Appl. Mater. Interfaces 2016, 8, 19843-19846.

(19) Weber, B.; Suhina, T.; Junge, T.; Pastewka, L.; Brouwer, A. M.; Bonn, D. Molecular Probes Reveal Deviations from Amontons' Law in Multi-Asperity Frictional Contacts. Nat. Commun. 2018, 9, 888.

(20) Suhina, T.; Weber, B.; Carpentier, C. E.; Lorincz, K.; Schall, P.; Bonn, D.; Brouwer, A. M. Fluorescence Microscopy Visualization of Contacts between Objects. Angew. Chem., Int. Ed. 2015, 54, 36883691.

(21) Ghosh, A.; Nakanishi, T. Frontiers of Solvent-Free Functional Molecular Liquids. Chem. Commun. 2017, 53, 10344-10357.

(22) Lakowicz, J. R. Principles of Fluorescence Spectroscopy; Springer, 2006.

(23) Hertz, H. Über Die Berührung Fester Elastischer Körper. J. Reine Angew. Math. 1882, 92, 156-171.

(24) Born, M.; Wolf, E. Principles of Optics; Pergamon Press: Liverpool, 1970.

(25) Jenkins, F. A.; White, H. E. Fundamentals of Optics, fourth ed.; McGraw-Hill, 1976.

(26) Jullien-Macchi, E.; Allain, C.; Alain-Rizzo, V.; Dumas-Verdes, C.; Galmiche, L.; Audibert, J. F.; Berhe Desta, M.; Pansu, R. B.; Audebert, P. New Highly Electrodeficient Cationic Fluorescent 
Tetrazines: A Step toward the Strongest Purely Organic Photooxidants. New J. Chem. 2014, 38, 3401-3407. 